\title{
Efecto in vitro del aceite de girasol (Helianthus annuus L.) sobre ácido transvacénico y patrón de ácidos grasos de cadena larga en la digesta ruminal
}

\author{
Juan Pablo Vélez-Ruiz ${ }^{(1)}{ }^{1}$, Elsa Beatriz Tequin-Ocampo (D) ${ }^{2}$, Julio Ernesto Vargas- \\ Sánchez \\ ${ }^{1}$ Joven investigador Colciencias. \\ 2 Estudiante de doctorado en Ciencias Agrarias, Universidad de Caldas.3Departamento de Producción Agropecuaria y Grupo de \\ Investigación CIENVET, Universidad de Caldas, Manizales, Colombia
}

jvargas@ucaldas.edu.co

Recibido: 23 de Septiembre de 2016 y Aprobado: 15 de Diciembre de 2016 y Actualizado: 15 de Junio de 2017

DOI: 10.17151/vetzo.2017.11.1.4

RESUMEN: La adición de aceites vegetales ricos en ácidos grasos de cadena larga (AGCL) a las dietas para rumiantes se ha propuesto como alternativa para disminuir las emisiones de metano $\left(\mathrm{CH}_{4}\right)$ y aumentar los niveles de ácido linoleico conjugado (ALC) en la leche y la carne, que está mediado por la producción a ácido transvacénico (ATV) en el rumen. El objetivo de este estudio fue evaluar el efecto de la adición de aceite de girasol en proporciones de $0 \%, 1 \%$ y 3\% (con base en MS) a substratos de fermentaciónin vitro (técnica de producción de gas): pasto Kikuyo (Pennisetum clandestinum) o pasto Ryegrass (Lolium perenne), sobre la cinética de producción de gas, la producción de $\mathrm{CH}_{4}$, la concentración de ácidos grasos volátiles (AGV), la producción de ALC y ATV y otros ácidos grasos de cadena larga (AGCL). La adición de aceite de girasol (1\% y 3\%) no disminuyó la producción de gas (A) ni la tasa de producción de gas (c); sin embargo, sí aumentó el tiempo de retardo en el inicio de la producción de gas $(0,042,0,425$ y $0,564 \mathrm{~h}$ para control, $1 \%$ y $3 \%$, respectivamente; $\mathrm{P}<0,001)$. La adición de aceites tampoco redujo la producción de CH4. No obstante, la adición de aceite incrementó ligeramente el porcentaje de ácido propiónico $(22,0,22,5$ y $22,6 \%$ para control, $1 \%$ y $3 \%$, respectivamente; $\mathrm{P}<0,05)$. La proporción de ácido linoleico conjugado (ALC) no varió como consecuencia de la adición de aceite, pero la proporción de ácido transvacénico (ATV) sí se incrementó significativamente en respuesta a la adición el aceite $(13,4,16 \%$ y $21,8 \%$ para control, $1 \%$ y $3 \%$, respectivamente; $\mathrm{P}<0,01)$. La inclusión de aceite de girasol a niveles del $1 \mathrm{y}$ $3 \%$ sobre la materia seca aumentó la producción de ATV a nivel ruminal sin afectar negativamente las variables que caracterizan la fermentación ruminal.

Palabras clave: ácido linoleico, fermentación ruminal, nutracéuticos, reducción de metano 


\title{
In vitro effect of sunflower oil (Helianthus Annuus L.) on transvacenic acid and long chain fatty acid pattern in ruminal digesta
}

\begin{abstract}
The addition of vegetable oils rich in long chain fatty acids (LCFA) to ruminant diets has been proposed as an alternative to reduce methane (CH4) and increase levels of conjugated linoleic acid (CLA) in milk and meat, that is preceded by the production of transvacenic acid (TVA) in the rumen. The objective of this study was to evaluate the effect of the supplementation of sunflower oil in proportions of $0 \%$, $1 \%$ and $3 \%$ (DM bases) to in vitro fermentation substrates (gas production technique):Pennisetum clandestinum or Lolium perenne, on the kinetics and total gas production, $\mathrm{CH} 4$ production, concentration of volatile fatty acids (VFA), production of CLA and TVA and the pattern of long chain fatty acids (LCFA). Addition of sunflower oil $(1 \%$ and $3 \%)$ did not decrease gas production $(\mathrm{A})$ or gas production rate (c); however, it increased the time delay to start fermentation (L, $0.042,0.425$ y $0.564 \mathrm{~h}$ for control, $1 \%$ y $3 \%$, respectively; $\mathrm{P}<0.001$ ). Oil addition also did not reduce production of $\mathrm{CH} 4$. Nevertheless, it slightly increased the percentage of propionic acid $(22,22.5 \mathrm{y}$ $22.6 \%$ for control, $1 \%$ y $3 \%$, respectively; $\mathrm{P}<0.05$ ). The proportion of conjugated linoleic acid (CLA) did not change because of the supplementation of oil, but the proportion of transvacenic acid (TVA) increased significantly in response to the addition of oil $(13.4,16 \%$ y $21.8 \%$ for control, $1 \%$ y $3 \%$, respectively; $\mathrm{P}<0.01)$. The inclusion of sunflower oil at levels of 1 and $3 \%$ of the dry matter increased ruminal production of TVA without affecting negatively the variables that characterize the ruminal fermentation.
\end{abstract}

Key words: linoleic acid, ruminal fermentation, nutraceuticals, reduction of methane

\section{Introducción}

En la actualidad se observa que los consumidores de alimentos de origen bovino muestran tendencia a substituir dichos alimentos por otros con menor cantidad de lípidos saturados y colesterol, y a considerar que esta fuente de lípidos conduce necesariamente a presentación de enfermedades cardiovasculares y a la tumorigénesis; sin embargo, un componente de este lípido, el ácido linoleico conjugado (ALC) ha mostrado actividad anticarcinogénica en ratas (Corl et al., 2001) y en humanos (Belury, 2002; Dilzer \& Park, 2012). También es un buen agente antiarteriosclerótico, antiinflamatorio, antidiabético y potenciador del sistema inmune (Dilzer \& Park, 2012) e inhibe la síntesis de ácidos grasos, reduciendo la acumulación de grasa corporal en individuos con sobrepeso (Weiss et al., 2004; McCrorie et al., 2011).

El ALC se origina en el rumen como resultado de la biohidrogenación incompleta del ácido linoleico y del ácido linolénico de origen alimentario (Kepler et al., 1966; Toral et al., 2016; Palmquist, 2007); aunque también puede sintetizarse endógenamente en la glándula mamaria a partir del ácido transvacénico (ATV) que también aparece en el rumen como producto de la biohidrogenación incompleta de los ácidos grasos C18 poliinsaturados (Corl et al., 2001; Gomez-Cortes et al., 2011; Prieto-Manrique et al., 
2016b). Por lo tanto, la presencia del ALC en los alimentos de origen bovino depende de las condiciones de alimentación de los animales (Mele et al., 2006; Prieto-Manrique et al., 2016a) y está estrechamente relacionada con la composición en ácidos grasos de los alimentos ingeridos. Se considera que los suplementos lipídicos con altos contenidos de ácido linoleico y alfa-linolénico (como los procedentes de semillas de soya, algodón, girasol, lino, cártamo y colza) son los más indicados para aumentar el ALC en leche (Khanal \& Dhiman, 2004).

Adicionalmente, se ha documentado que en los rumiantes el aumento en el consumo de dichos ácidos grasos es ambientalmente relevante debido a que pueden inducir la disminución en la producción de metano $\left(\mathrm{CH}_{4}\right)$ ruminal (Beauchemin et al., 2009; Jalc et al., 2007; Toprak, 2015) hasta en un 50\% (Machmüller et al., 1998), y que la depresión en la producción de $\mathrm{CH}_{4}$ es mayor cuando aumentan las instauraciones en los ácidos grasos C:18 (Cosgrove et al., 2008). El interés por reducir la emisión de metano está dado por su mayor potencia para generar calentamiento global (21 veces mayor que el $\mathrm{CO}_{2}$ ) (IPCC, 2007) y porque constituye entre 50 a $80 \%$ de la huella de carbono de los alimentos provenientes de los rumiantes (Moss et al., 2000; Flachowsky \& Kamphues, 2012); también porque éste constituye una pérdida de la energía consumida de entre 4 y $10 \%$ con extremos de 2 a 15\% (Patra \& Yu, 2013).

El objetivo del estudio fue determinar el efecto de la adición de aceite de girasol (Helianthus annuus L.) sobre la producción de ALC y de ATV, y de $\mathrm{CH}_{4}$ en las condiciones de ambiente ruminal provocadas por pastos propios de trópico frío.

\section{Materiales y Métodos}

El trabajo se desarrolló usando la técnica de producción de gas (Elghandour et al., 2015; López et al., 2007; Theodorou et al., 1994).

Substrato de fermentación: se utilizaron como substratos de fermentación dos pastos de trópico frío: Kikuyo (Pennisetum clandestinum) y Ryegrass (Lolium perenne); de cada uno de ellos se recolectaron tres muestras independientes $(500 \mathrm{~g})$, en estado de prefloración y usando un método de muestreo que imitaba en la altura el patrón de consumo exhibido por los animales en los lotes aledaños recién pastoreados.

La mitad de cada muestra $(250 \mathrm{~g})$ de pasto fue picada $(5 \mathrm{~cm})$, congelada $\left(-26^{\circ} \mathrm{C}\right)$, liofilizada, molida $(1 \mathrm{~mm})$ y usada como substrato de fermentación; la otra porción (250 g) fue usada para determinar materia seca (MS) mediante secado en horno (marca MRC de la serie DNI-50) a $60^{\circ} \mathrm{C}$ con pesajes cada 24 horas hasta obtener peso constante y luego fue sometido al análisis bromatológico mediante las técnicas analíticas convencionales de la AOAC (AOAC, 1999) (MS método ID 934.01, proteína bruta método ID 984.13 y fibra detergente neutro (Van Soest et al., 1991).

Tratamientos: los tratamientos estuvieron determinados por el forraje y la proporción de aceite de girasol: $1 \%$ y $3 \%$ de la MS o sin adición, así: 
1. Kikuyo (Pennisetum clandestinum) sin aceite

2. Kikuyo (Pennisetum clandestinum) con 1\% de aceite de girasol

3. Kikuyo (Pennisetum clandestinum) con 3\% de aceite de girasol

4. Ryegrass (Lollium perenne) sin aceite

5. Ryegrass (Lollium perenne) con $1 \%$ de aceite de girasol

6. Ryegrass (Lollium perenne) con 3\% de aceite de girasol

El aceite de girasol comercial se mezcló con los forrajes $24 \mathrm{~h}$ antes del inicio de la fermentación. Luego se puso el substrato de fermentación $(0,5 \mathrm{~g}$ de MS) dentro de botellas (FIC-5 de $100 \mathrm{ml}$ ) previamente rotuladas, que fueron almacenadas a $-4^{\circ} \mathrm{C}$ hasta iniciar la inoculación. Las botellas se prepararon por cuadruplicado: dos de estas botellas se incubaron por 144 horas para determinar la cinética de fermentación, las otras dos botellas se incubaron por espacio de 24 horas para determinar el perfil de ácidos grasos de cadena larga (AGCL) y los ácidos grasos volátiles (AGV).

Diseño experimental y análisis estadístico: El diseño experimental utilizado fue el completamente al azar con arreglo factorial 2 x 3 (dos pastos y tres niveles de adición de aceite: 0,1 y $3 \%$ de la MS).

El modelo de análisis estadístico fue el siguiente:

$\mathrm{y} \ddot{\boldsymbol{j}} \boldsymbol{k}=\mu+\alpha \boldsymbol{i}+\beta \boldsymbol{j}+\alpha \beta(\boldsymbol{i} \boldsymbol{j})+\varepsilon \boldsymbol{i} \boldsymbol{j} \boldsymbol{k}$,

donde yijk es cada observación individual, $\boldsymbol{\mu}$ es la media, $\boldsymbol{\alpha} \boldsymbol{i}$ es el efecto del factor pasto (Kikuyo o Ryegrass), $\boldsymbol{\beta} \boldsymbol{j}$ es el efecto del nivel de inclusión de aceite, $\boldsymbol{\alpha} \boldsymbol{\beta}(\boldsymbol{i j})$ es la interacción entre ambos factores y $\varepsilon i j k$ es la varianza o error residual.

Fuente del inóculo de fermentación: la fuente del inóculo de fermentación (líquido ruminal) se obtuvo de dos bovinos cebú provistos de una cánula ruminal (Preston, 1995) y pastoreando una pradera de Kikuyo y Ryegrass (75\% y 25\%), solo se suplementó con sal mineralizada. El inóculo se obtuvo en horas de la mañana (rumen lleno) mediante la extracción manual del material pastoso presente en la porción ventral caudal y ventral craneal de la cavidad ruminal, dicho material se filtró usando gasa aséptica y se recogió el líquido resultante en recipientes isotérmicos de $2 \mathrm{~L}$ para su trasladado al laboratorio.

En laboratorio, el líquido ruminal se filtró una vez más, se dispuso en vasos de precipitado $(1 \mathrm{~L})$ y se mantuvo a $39^{\circ} \mathrm{C}$ y gaseado con $\mathrm{CO}_{2}$ hasta el momento de la inoculación.

Medio de cultivo: la preparación del medio de cultivo se hizo utilizando una mezcla de diluciones de macrominerales y microminerales, dilución buffer, dilución reductora y resazurina en un medio anaerobio conseguido mediante la saturación de la mezcla con CO2 (Menke \& Steingass, 1988).

La dilución de macrominerales se preparó con 4,2 $\mathrm{g}$ de fosfato de sodio anhidro $\left(\mathrm{Na}_{2} \mathrm{HPO}_{4}-\mathrm{Lab}\right.$. CARLO ERBA), 4,6 g de fosfato de potasio anhidro $\left(\mathrm{KH}_{2} \mathrm{PO}_{4}-\mathrm{Lab}\right.$. CARLO ERBA) y 0,4 g de sulfato de magnesio $\left(\mathrm{MgSO}_{4} 7 \mathrm{H}_{2} \mathrm{O}-\mathrm{Lab}\right.$. CARLO ERBA) en $741,1 \mathrm{ml}$ de agua destilada. La dilución de microminerales estuvo compuesta por 0,049 $\mathrm{g}$ de cloruro de calcio $\left(\mathrm{CaCl}_{2} 2 \mathrm{H}_{2} \mathrm{O}-\mathrm{Lab}\right.$. CARLO ERBA), 0,037 $\mathrm{g}$ de cloruro de manganeso $\left(\mathrm{MnCl}_{2} 2 \mathrm{H}_{2} \mathrm{O}\right.$ - Lab. MERCK), $0,004 \mathrm{~g}$ de cloruro de cobalto $\left(\mathrm{CoCl}_{2} 6 \mathrm{H}_{2} \mathrm{O}\right.$ 
- Lab. SCHARLAU) y 0,030 g de cloruro de hierro (FeCl3 6H2O - Lab. MERCK) en $3,7 \mathrm{ml}$ de agua destilada.

La solución buffer fue preparada a partir de $0,004 \mathrm{~g}$ de carbonato de amonio $\left(\left(\mathrm{NH}_{4}\right)\right.$ $\mathrm{HCO}_{3}-\mathrm{Lab}$. CARLO ERBA), y 0,218 g de bicarbonato de sodio $\left(\mathrm{NaHCO}_{3}-\mathrm{Lab}\right.$. CARLO ERBA) en $741 \mathrm{ml}$ de agua destilada. La resazurina se mezcló a razón de 0,004 $\mathrm{g}\left(\mathrm{Cl}_{2} \mathrm{H}_{6} \mathrm{NNaO}_{4}-\mathrm{Lab}\right.$. PANREAC) con $3,7 \mathrm{ml}$ de agua destilada.

Finalmente se elaboró la dilución reductora, compuesta de 0,926 g de hidrocloruro de cisteína $\left(\mathrm{C}_{3} \mathrm{H}_{8} \mathrm{CINO}_{2} \mathrm{~S} * \mathrm{H}_{2} \mathrm{O}-\mathrm{Lab}\right.$. MERCK), 5,929 $\mathrm{ml}$ de hidróxido de sodio (1N) (NaOH - Lab. CARLO ERBA) y 0,926 g de sulfuro de sodio $\left(\mathrm{Na}_{2} \mathrm{~S}_{9} \mathrm{H}_{2} \mathrm{O}\right.$ - Lab. CARLO ERBA) en 148,2 ml de agua destilada. Para su preparación se usó una botella de vidrio de $5 \mathrm{~L}$ puesta sobre una placa de calentamiento a $39^{\circ} \mathrm{C}$ con agitación magnética (WISD - MSH-20D) y sometida a gaseado con $\mathrm{CO}_{2}$.

Luego de tener la solución reductora homogenizada y a $39^{\circ} \mathrm{C}$, se adicionaron los macrominerales, microminerales, resazurina y dilución buffer; y se mantuvo en la placa de calentamiento con agitación y gaseado con $\mathrm{CO} 2$ hasta que el medio de cultivo tomó un color rosado brillante que indica su saturación con el gas (aprox. 2 h).

Inoculación: poco antes de la incubación los frascos con substrato se precalentaron a $39^{\circ} \mathrm{C}$ usando la incubadora. Luego mediante el uso de una jeringa a cada frasco se le adicionó $40 \mathrm{ml}$ de medio de cultivo y $10 \mathrm{ml}$ de líquido ruminal, se gaseó con $\mathrm{CO}_{2}$ y luego se selló con el tapón de caucho y el agrafe de aluminio. Después se picharon las botellas (aguja calibre 20 de 1 pulgada), para equilibrar las presiones interna y externa, se agitaron con suavidad y se ubicaron en bandejas metálicas dentro de la incubadora $\left(39^{\circ} \mathrm{C}\right)$.

Muestreos: como se mencionó, dos de las botellas preparadas por cuadruplicado se incubaron por 144 horas, a éstas se les realizó un seguimiento de la producción de gas y de la presión (PSI) a las 3, 6, 9, 12, 16, 20, 24, 29, 34, 44, 56, 68, 83, 98, 120 y 144 horas post incubación. En cada ocasión se registró la hora de inicio y de finalización de la medición. Para la medición de presión se usó un equipo DELTA -2304-0, equipado con una sonda de presión relativa DTP-704-2BGI, que se acopló a una llave de tres vías a la cual también se acoplaron una aguja (referencia 20G de 1 pulgada), para pinchar a través de los tapones de los frascos y obtener la lectura de la presión, y una jeringa de $(20 \mathrm{ml})$ de para extraer el gas y determinar su volumen. El gas obtenido en cada lectura se fue depositando dentro de bolsas herméticas hasta la lectura de $48 \mathrm{~h}$, momento en que se tomó una muestra de gas $(12 \mathrm{ml})$ y se depositó en tubos de $10 \mathrm{ml}$ con vacío (VACUETTE) hasta la realización del análisis de $\mathrm{CH}_{4}$. Luego de obtener las lecturas de presión y volumen de gas, las botellas se agitaron y luego se regresaron a la incubadora hasta la siguiente lectura.

Con la segunda pareja de botellas preparadas por cuadruplicado se siguió este mismo procedimiento para la lectura de presión y obtención de gas, pero solo durante las primeras $24 \mathrm{~h}$ de incubación. En ese momento las botellas fueron destapadas para obtener una muestra de su contenido $(40 \mathrm{ml}$ ), que se depositó en tubos FALCON (marca Fisher Brand) de $50 \mathrm{ml}$, donde se mantuvo hasta la realización del análisis de ácidos grasos de cadena larga (AGCL, incluidos ALC y ATV). También se obtuvo una muestra de $3 \mathrm{ml}$ de contenido de cada botella que se depositó en tubos de ensayo de $6 \mathrm{ml}$ 
(VACUETTE) y se usó para el análisis de ácidos grasos volátiles (AGV). Tanto las muestras para análisis de AGCL como las muestras para AGV se conservaron a $-20^{\circ} \mathrm{C}$; las muestras de gas se conservaron a temperatura ambiente.

Determinación de la cinética de producción de gas: se hizo a partir de los datos de producción acumulada de gas previa corrección por blancos y tiempo de incubación, los datos se ajustaron (individualmente para cada botella) al modelo exponencial:

$\mathrm{G}=\mathrm{A}\left(1-\mathrm{e}^{-\mathrm{c}(\mathrm{t}-\mathrm{L})}\right)$

donde:

"G" es la producción acumulada de gas (ml)

"A" es la producción de gas a partir de la fermentación $(\mathrm{ml})$

" $c$ " es la tasa de producción de gas

" $\boldsymbol{L}$ " (Lag) es el tiempo en horas antes de iniciar la producción de gas.

Para el ajuste de las curvas de producción de gas se utilizó el programa SAS.

Determinación de metano: en las muestras de gas se determinó la concentración de $\mathrm{CH}_{4}$ mediante cromatografía de gases, para ello se usaron una columna STABILWAXDA con límites de temperatura: 40 a $240 / 250^{\circ} \mathrm{C}$ longitud: $30 \mathrm{~m}$ ID: $0,53 \mathrm{~mm} \mathrm{df}(\mu \mathrm{m})$ : $1.00 \mu \mathrm{m}$, marca RESTEK; y un detector de ionización de llama FID Marca Agilent HP modelo 3890, el gas de arrastre (helio) tuvo un flujo de $24 \mathrm{ml} / \mathrm{min}$. El contenido de $\mathrm{CH}_{4}$ se determinó mediante calibración externa, usando una mezcla de gases certificada. Las pruebas se desarrollaron usando un equipo de cromatografía de gases-masas marca Shimadzu GC Modelo 2010, con un inyector Shimadzu AOC - 20i y un espectrómetro Shimadzu GCMSQP2010.

Determinación de ácidos grasos de cadena larga: para el análisis de ácidos grasos de cadena larga del líquido ruminal, se llevaron a cabo los siguientes procedimientos:

1. Extracción: luego de liofilizar $40 \mathrm{ml}$ de la muestra de líquido ruminal se tomaron $500 \mathrm{mg}$, se sometieron a extracción utilizando cloroformo y metanol (2:1) (Bligh \& Dyer, 1959) y se obtuvieron los ácidos grasos utilizando un rotavapor.

2. Derivatización: la volatilización de los ácidos grasos (formación de su metiléster), necesaria para su análisis mediante cromatografía de gases, se hizo utilizando trifluoro de boro en metanol (ácido de Lewis) (Christie, 1990).

3. Cromatografía de gases: el análisis de AGCL se realizó por cromatografía de gases acoplado a espectrometría de masas, empleando una columna CP-Sil 88 de $100 \mathrm{~m}$. La identificación de los AGCL se hizo utilizando el estándar FAME mix 37 de Supelco y los estándares en forma ácida de ALC y ATV (Gomez-Cortes et al., 2008).

Determinación de ácidos grasos volátiles $(\boldsymbol{A G V})$ : para hacer la determinación de AGV (acético, propiónico, butírico), se empleó un total de $0,8 \mathrm{ml}$ de la muestra de líquido ruminal dispuesta para ello; se preparó para un análisis de cromatografía de gases, mediante la adición de $0,5 \mathrm{ml}$ de una solución con $20 \mathrm{~g} / \mathrm{L}$ de ácido metafosfórico y 4 $\mathrm{g} / \mathrm{L}$ de ácido crotónico (usado como estándar interno) en ácido clorhídrico 0,5 $\mathrm{N}$. Adicionalmente, se usó un estándar externo con los ácidos: acético, propiónico, butírico, isobutírico, pentanoico, isopentanoico y hexanoico, este se usó como patrón externo con 
el cual se estableció la curva de calibración de cada AGV. El análisis se hizo usando cromatografía de gases con detector FID con una columna semicapilar, TR-FFAP, $30 \mathrm{~m}$ $\times 0,53 \mathrm{~mm} \times 1 \mathrm{~m}($ Supelco, USA) (García-González et al., 2008).

\section{Resultados y Discusión}

Contenido de PB y FDN en los pastos: el pasto Kikuyo presentó niveles de proteína bruta (PB) de 19,4\% y de fibra detergente neutra (FDN) de 55\%, correspondiéndose con la literatura, donde se registran valores de PB de $15,4 \%$ a 27,1\% y de FDN de $51,7 \%$ a 66,9\% (Correa et al., 2009). El Ryegrass tuvo PB de 19,7\% y FDN de 49,7\%, valores ligeramente superiores a los presentados por Estrada (2002), quien registra valores de PB y FDN de 16,9\% y 45,7\%, respectivamente (Tabla 1 ).

Tabla 1. Análisis del pasto Kikuyo (Pennisetum clandestinum) y del pasto Ryegrass (Lolium perenne).

\begin{tabular}{lccc}
\hline Pasto & $\begin{array}{c}\text { Nitrógeno total } \\
(\mathbf{\%})\end{array}$ & $\begin{array}{c}\text { Proteína bruta } \\
\mathbf{( \% )}\end{array}$ & $\begin{array}{c}\text { FDN } \\
\mathbf{( \% )}\end{array}$ \\
\hline Kikuyo (Pennisetum clandestinum) & 3,1 & 19,3 & 55,0 \\
Ryegrass (Lolium perenne) & 3,2 & 19,7 & 49,7 \\
\hline FDN= fibra detergente neutra & \multicolumn{3}{|}{}
\end{tabular}

Cinética de fermentación: Los parámetros de la cinética de producción de gas se presentan en la Tabla 2. En el caso de los pastos, se observaron diferencias significativas $(\mathrm{P}<0,001)$ para todas las variables: producción máxima de gas $(\mathrm{A})$, tasa de fermentación (c) y tiempo de retardo (L). El pasto Kikuyo fue el forraje de mayor producción acumulada de gas (A). Una elevada producción de gas está asociada principalmente a un alto contenido de proteína bruta, un moderado contenido de FDN (inferior al 60\%) y una baja proporción de lignina (Sánchez et al., 2005) (Li \& Meng, 2006). Sin embargo, el Kikuyo tuvo menor tasa de fermentación (c) lo cual puede estar asociado a su mayor contenido de FDN, y a una correspondiente y eventual menor proporción de carbohidratos no estructurales (CNE) de fácil fermentación (Sánchez, Arreaza \& Abadía, 2005); se ha registrado que el pasto Kikuyo contiene 13,4\% de CNE, que frente a $22 \%$ de pastos como el Brachiaria decumbens, le hacen un sustrato de más lenta tasa de fermentación (Correa, Pabón \& Carulla, 2009). Contrario a lo esperado dada su calidad, con un contenido de FDN inferior al pasto Kikuyo, el pasto Ryegrass produjo menos gas. Sin embargo, su tasa de fermentación fue notablemente mayor $(0,057$ vs 0,038$)$ y tuvo menor tiempo de demora antes del inicio de la degradación. Lo que podría indicar que en esta pastura la colonización bacteriana del substrato es más eficiente. 
Tabla 2. Parámetros de la curva de producción de gas in vitro.

\begin{tabular}{|c|c|c|c|}
\hline & $\begin{array}{c}\mathrm{A} \\
\mathrm{ml} / \mathrm{g} \mathrm{MS}\end{array}$ & c & $\begin{array}{c}\mathrm{L} \\
\text { (horas) }\end{array}$ \\
\hline \multicolumn{4}{|l|}{ Pastos } \\
\hline Kikuyo & 322,5 & 0,038 & 0,619 \\
\hline Ryegrass & 302,2 & 0,057 & 0,069 \\
\hline$P \leq$ & 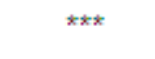 & 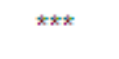 & 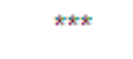 \\
\hline DMS & 3,77 & 0,001 & 0,097 \\
\hline \multicolumn{4}{|l|}{ Aceite } \\
\hline Aceite girasol $0 \%$ & 312,0 & 0,046 & 0,042 \\
\hline Aceite girasol $1 \%$ & 312,2 & 0,049 & 0,425 \\
\hline Aceite girasol $3 \%$ & 313,0 & 0,048 & 0,564 \\
\hline$P \leq$ & NS & * & $\star \star \star$ \\
\hline DMS & 4,61 & 0,001 & 0,119 \\
\hline
\end{tabular}

Respecto a la adición de aceite de girasol, no se observaron diferencias entre los tres niveles de inclusión $(0 \%, 1 \%$ y $3 \%$ ) en cuanto a la variable de producción de gas (A), es decir que la adición de aceite no afectó negativamente la fermentación. Lo cual corresponde con el hecho que el efecto negativo de las grasas sobre la fermentación depende del tipo de grasa y con niveles de grasa superiores al 6\% MS de dieta (Broudiscou et al., 1994). Hervás et al. (2001), tampoco observaron in vitro efecto negativo de bajos niveles de suplementación lipídica (1 y 2\%) e incluso observaron aumento con el $2 \%$ de adición de aceite. Por otro lado, la adición de aceite sí alteró $(\mathrm{P}<0,05)$ la tasa de producción de gas $(\mathrm{c})$ : fue mayor con el $1 \%$ de aceite de girasol, seguido por el de 3\% de aceite y menor en el control sin aceite; Hervás et al. (2001) también observaron alteración de la tasa de producción de gas debido a la adición de aceite $(34,5 \mathrm{ml} / \mathrm{h}, 30,6 \mathrm{ml} / \mathrm{h}$ y $40,3 \mathrm{ml} / \mathrm{h}$ con niveles de adición de 0,150 y 300 mililitros de grasa en 14 gramos de pasto). Al mismo tiempo se observó un aumento significativo $(\mathrm{P}<0,001)$ en el tiempo de demora para el inicio de la producción de gas: la adición de aceite hizo que la demora pasara de $0,04 \mathrm{~h}$ a $0,42 \mathrm{~h}$ y $0,56 \mathrm{~h}$ con la adición de aceite al 1 y $3 \%$, respectivamente, efecto que también ha sido registrado con anterioridad (Arenas et al., 2010; Patra \& Yu, 2013).

Producción de metano $\left(\mathrm{CH}_{4}\right)$ : la producción total y el porcentaje de $\mathrm{CH}_{4}$ en relación al gas producido a 24 y 48 horas de fermentación in vitro se muestran en la Tabla 3. Solo se observaron diferencias significativas $(\mathrm{P}<0,01)$ en la producción y en el porcentaje de $\mathrm{CH}_{4}$ a las $24 \mathrm{~h}$ postincubación. En este tiempo, el pasto Ryegrass produjo mayor cantidad de metano $(27,2 \mathrm{ml} / \mathrm{g}$ MS) que el pasto Kikuyo, pero ambos pastos tuvieron igual concentración de $\mathrm{CH}_{4}$; por lo tanto, la mayor producción de metano del pasto Ryegrass a las 24 horas de incubación estuvo determinada por su considerablemente mayor tasa de producción gas que la del pasto Kikuyo, que determina a su vez una mayor producción de gas total a las 24 horas de incubación. Sin embargo, se ha estimado que las gramíneas C4 (Kikuyo) producen más metano (hasta 14,3\%) por 
unidad de energía digestible que las especies de clase C3 (Ryegrass) y esto se atribuye a una mayor proporción de celulosa y lignina en las primeras (Vargas et al., 2012).

Tabla 3. Producción de metano ( $\mathrm{ml} / \mathrm{g}$ MS y \%) a las 24 y 48 horas de incubación.

\begin{tabular}{|c|c|c|c|c|}
\hline & $\begin{array}{c}\mathrm{CH}_{4}-24 \mathrm{~h} \\
\mathrm{ml} / \mathrm{g} \mathrm{MS}\end{array}$ & $\begin{array}{c}\mathrm{CH}_{4}-48 \mathrm{~h} \\
\mathrm{ml} / \mathrm{g} \mathrm{MS}\end{array}$ & $\begin{array}{c}\% \mathrm{CH}_{4} \\
24 \mathrm{~h}\end{array}$ & $\begin{array}{c}\% \mathrm{CH}_{4} \\
48 \mathrm{~h}\end{array}$ \\
\hline \multicolumn{5}{|l|}{ Pasto } \\
\hline Kikuyo & 22,8 & 61,3 & 12,0 & 22,8 \\
\hline Ryegrass & 27,2 & 58,5 & 12,1 & 20,6 \\
\hline$P \leq$ & $\star \star \star ~$ & NS & NS & NS \\
\hline DMS & 0,63 & 1,25 & 1,52 & 4,20 \\
\hline \multicolumn{5}{|l|}{ Aceite } \\
\hline Aceite girasol $0 \%$ & 22,5 & 58,4 & 11,0 & 21,2 \\
\hline Aceite girasol $1 \%$ & 27,1 & 62,3 & 12,8 & 22,4 \\
\hline Aceite girasol $3 \%$ & 25,4 & 59,0 & 12,5 & 21,6 \\
\hline$P \leq$ & $*$ & NS & * & NS \\
\hline DMS & 0,77 & 1,53 & 1,87 & 5,15 \\
\hline
\end{tabular}

Por otra parte y contrario a lo esperado, la adición de niveles del 1 y $3 \%$ de aceite de girasol incrementó ligeramente la producción $(22,5,27,1$ y 25,4 ml/g MS para $0 \% 1 \%$ y $3 \%$ de aceite, respectivamente) y la concentración de $\mathrm{CH} 4(11,12,8$ y $12,5 \%$ para $0 \%$ $1 \%$ y $3 \%$ de aceite, respectivamente). La adición de lípidos puede reducir la emisión deCH4 ruminal, disminuyendo: la cantidad de materia orgánica fermentada en el rumen, la actividad de las bacterias metanogénicas o el número de protozoos (Cieslak et al., 2013; Patra \& Yu, 2013); y a través del uso de hidrógeno durante el proceso de biohidrogenación (Johnson \& Johnson, 1995), pudiendo alcanzar hasta un 50\% de reducción (Machmüller et al., 1998). Efectivamente, contrario a lo encontrado en este trabajo se han registrado reducciones substanciales en la producción de CH4 a través de la suplementación de aceites, especialmente aceites ricos en ácidos grasos de cadena media ( $\leq \mathrm{C}: 14)$ y de cadena larga ( $>\mathrm{C}: 14$ ) (Fievez et al., 2003; Jordan et al., 2006). La suplementación con ácidos grasos de cadena larga $(\mathrm{C}: 18)$ ha generado efectos antimetanogénicos (Jalc et al., 2007; Beauchemin et al., 2009). Sin embargo, Cosgrove et al. (2008) encontraron que la infusión de aceites al rumen en concentraciones inferiores al 5\% no afectó la producción de CH4. Incluso con niveles de hasta $10 \%$ de suplementación de aceite de girasol en la dieta, solo se ha observado una ligera disminución en la producción de CH4 (Soder et al., 2013).

En términos generales, el porcentaje de $\mathrm{CH}_{4}$ a las $48 \mathrm{~h}$ es 1,8 veces mayor que a las $24 \mathrm{~h}$ de incubación; según Abrego (2012) en los sistemas de cultivo cerrado siempre hay un nutriente esencial que se agota a través del tiempo y entonces se altera la fermentación y acumulación de los productos de desecho; tras la hidrólisis de los polímeros del sustrato las bacterias utilizan casi todo el hidrógeno en la producción de $\mathrm{CH}_{4}$, lo que indica dominio de las bacterias metanogénicas en el ecosistema ruminal (Chaucheyras-Durand et al., 2010). 
Ácidos grasos volátiles $(A G V)$ : la concentración de los ácidos grasos volátiles se presenta en la Tabla 4. Respecto al nivel de adición de aceite, no se observaron diferencias en las concentraciones (mg/L) de AGV; sin embargo, se detectaron ligeras diferencias en la proporción de ácido propiónico entre los tratamiento con y sin aceite. Otros trabajos han mostrado que la utilización de diversos aceites (coco, girasol, pescado y almendras) no ha afectado las proporciones de AGV, aunque sí es posible ver cambios en la relación acético/propiónico (Panyakaew et al., 2013).

Tabla 4. Ácidos grasos volátiles ( $\mathrm{mg} / \mathrm{L}$ y \%) en el medio de cultivo a las 24 horas de incubación.

\begin{tabular}{|c|c|c|c|c|c|c|}
\hline & \multicolumn{2}{|c|}{ Acético } & \multicolumn{2}{|c|}{ Propiónico } & \multicolumn{2}{|c|}{ Butírico } \\
\hline & $\mathrm{mg} / \mathrm{L}$ & $\%$ & $\mathrm{mg} / \mathrm{L}$ & $\%$ & $\mathrm{mg} / \mathrm{L}$ & $\%$ \\
\hline \multicolumn{7}{|l|}{ Pasto } \\
\hline Kikuyo & 4191,2 & 66,1 & 1423,9 & 22,4 & 723,6 & 11,4 \\
\hline Ryegrass & 4126,6 & 65,1 & 1415,3 & 22,3 & 792,8 & 12,6 \\
\hline$P \leq$ & NS & $* \star \star *$ & NS & NS & NS & $* \star \star *$ \\
\hline DMS & 245,6 & 0,23 & 87,4 & 0,17 & 41,4 & 0,25 \\
\hline \multicolumn{7}{|c|}{ Aceite de Girasol } \\
\hline $0 \%$ & 4185,2 & 65,8 & 1401,5 & 22,0 & 768,2 & 12,1 \\
\hline $1 \%$ & 4243,1 & 65,7 & 1453,5 & 22,5 & 764,1 & 11,8 \\
\hline $3 \%$ & 4048,5 & 65,3 & 1403,8 & 22,6 & 742,2 & 12,1 \\
\hline$P \leq$ & NS & NS & NS & $\star$ & NS & NS \\
\hline DMS & 300,7 & 0,29 & 106,9 & 0,21 & 50,7 & 0,31 \\
\hline
\end{tabular}

Respecto a la pastura, se encontró que el Kikuyo genera una proporción de acético ligeramente mayor $(66,1 \%$ vs $65,1 \%)$, lo cual corresponde con un mayor contenido de FDN (Correa, Pabón \& Carulla, 2009); en contraste, el Ryegrass produjo un nivel superior de butírico $(12,6 \%$ vs $11,4 \%)$, efecto que también fue observado por Hervás et al. (2001). El aumento en la producción de CH4 está generalmente acompañado de un aumento en la relación acético-propiónico (McAllister \& Newbold, 2008; Lila et al., 2005; Russell, 1998); en el presente estudio aunque la producción de $\mathrm{CH}_{4}$ a las $24 \mathrm{~h}$ fue diferente entre las pasturas, la relación acético/propiónico fue similar.

Ácidos grasos de cadena larga (AGCL): la proporción de ácidos grasos de cadena larga en la digesta (postincubación) se muestra en la Tabla 5. La inclusión de niveles cada vez mayores de aceite de girasol generó un aumento significativo $(\mathrm{P}<0,01)$ en la proporción ATV en la digesta. Con una inclusión de 3\% de aceite de girasol el ATV alcanzó una proporción de 21,8\%; incluso la adición de $1 \%$ de aceite de girasol aumentó la proporción de ATV, pero en menor medida (16\%, vs 13,45 en el control), lo que corresponde con el efecto normalmente esperado (Khanal \& Dhiman, 2004; Jayanegara et al., 2012). No se registraron efectos de la adición de aceite sobre la concentración de ALC; sin embargo, el notorio efecto observado sobre la proporción de ATV que se presentó como consecuencia de la adición de aceite y el hecho que gran parte del 
isómero ALC presente en la leche es sintetizado a partir del ATV formado en el rumen (Bauman et al., 2000), permite pensar que la metodología empleada es apropiada para evaluar el potencial de diferentes fuentes y niveles de suplementación lipídica sobre la producción de ATV a nivel ruminal, en perspectiva de mejorar los niveles de ALC en la carne y la leche de los rumiantes, como se ha observado en estudios in vivo (AbuGhazaleh (2008).

La adición de aceite de girasol también alteró la proporción de los ácidos: palmítico $(\mathrm{P}<0,05)$, oleico $(\mathrm{P}<0,001)$ y linolénico $(\mathrm{P}<0,01)$ : aumentó la proporción relativa del ácido oleico y al mismo tiempo disminuyó la proporción de los ácidos palmítico, linoleico y linolénico. En relación con las pasturas, no se observaron diferencias en las proporciones de ATV o ALC, ni en los ácidos palmítico, esteárico, oleico, linoleico y linolénico.

Tabla 5. Perfil de ácidos grasos de cadena larga (\%).

\begin{tabular}{|c|c|c|c|c|c|c|c|c|c|}
\hline & $\begin{array}{c}\text { Past } \\
0\end{array}$ & & & Aceit & $\mathrm{ede}$ & irasol & & & \\
\hline & $\begin{array}{c}\text { Kikuy } \\
\circ\end{array}$ & $\begin{array}{c}\text { Ryegras } \\
\mathrm{s}\end{array}$ & $P \leq$ & $\begin{array}{l}\mathrm{DM} \\
\mathrm{S}\end{array}$ & $0 \%$ & $1 \%$ & $3 \%$ & $P \leq$ & $\begin{array}{l}\text { DM } \\
S\end{array}$ \\
\hline Palmítico & 16,0 & 15,2 & $\begin{array}{c}0,39 \\
7\end{array}$ & 0,92 & 17,6 & 15,4 & 13,9 & * & 1,12 \\
\hline Esteárico & 56,7 & 54,4 & $\begin{array}{c}0,44 \\
5\end{array}$ & 2,95 & 56,6 & 57,1 & 53,0 & $\begin{array}{c}0,49 \\
2\end{array}$ & 3,61 \\
\hline Transvacénico & 17,7 & 16,5 & $\begin{array}{c}0,52 \\
4\end{array}$ & 1,80 & 13,4 & 16,0 & 21,8 & $\star * *$ & 2,21 \\
\hline NN1 ${ }^{a}$ & 1,5 & 3,5 & $\star \star \star \star$ & 0,35 & 2,7 & 2,4 & 2,3 & $\begin{array}{c}0,65 \\
9\end{array}$ & 0,43 \\
\hline Oleico & 1,3 & 1,8 & $\begin{array}{c}0,28 \\
8\end{array}$ & 0,41 & 0,54 & 1,2 & 2,8 & $\star \star \star *$ & $\begin{array}{c}0,50 \\
3\end{array}$ \\
\hline $\mathrm{NN} 2^{\mathrm{b}}$ & 0,07 & 0,51 & $\star *$ & 0,12 & 0,50 & 0,3 & 0,05 & $\begin{array}{c}0,02 \\
8\end{array}$ & $\begin{array}{c}0,14 \\
5\end{array}$ \\
\hline NN $3^{c}$ & 1,3 & 2,4 & * & 0,47 & 2,4 & 1,7 & 1,57 & $\begin{array}{c}0,36 \\
4\end{array}$ & 0,57 \\
\hline
\end{tabular}




\begin{tabular}{|c|c|c|c|c|c|c|c|c|c|}
\hline \multirow[t]{2}{*}{ Linoleico } & 1,5 & 1,6 & 0,81 & 0,22 & 1,7 & 1,6 & 1,42 & 0,54 & 0,26 \\
\hline & & & 2 & & & & & 0 & \\
\hline \multirow[t]{2}{*}{$\mathrm{NN} 4^{\mathrm{d}}$} & 0,58 & 0,71 & 0,64 & 0,26 & 0,67 & 0,67 & 0,58 & 0,93 & 0,31 \\
\hline & & & 2 & & & & & 4 & 6 \\
\hline \multirow[t]{2}{*}{ Linolénico } & 2,8 & 2,9 & 0,95 & 0,36 & 3,9 & 2,7 & 2,0 & $\star *$ & 0,44 \\
\hline & & & 0 & & & & & & \\
\hline C18:2 c9t11 & 0,641 & 0,588 & 0,90 & 0,43 & 0,69 & 0,62 & 0,52 & 0,94 & 0,53 \\
\hline ALC & & & 4 & 3 & 5 & 8 & 0 & 6 & 1 \\
\hline $\mathrm{C} 18: 2 \mathrm{t} 10 \mathrm{c} 12$ & 0,067 & 0,032 & 0,46 & 0,04 & 0,03 & 0,04 & 0,06 & 0,81 & 0,05 \\
\hline ALC & & & 2 & 5 & 3 & 7 & 8 & 9 & 6 \\
\hline
\end{tabular}

\section{Conclusiones}

La inclusión de aceite de girasol en el sustrato de fermentación (pasturas de trópico frío) aumentó notoria y significativamente la proporción relativa de ATV, pero no afectó la de ALC. El efecto sobre el ATV fue mayor con la adición de 3\% de aceite. Excepto un leve aumento en el tiempo de inicio de la producción gas, la adición de aceite no tuvo un efecto negativo sobre la fermentación: tasa y producción de gas ni sobre la proporción de $\mathrm{AGV}$. La adición de aceites también aumentó la proporción de ácido oleico, pero disminuyó las proporciones de los ácidos palmítico, linoleico y linolénico. La tasa de producción de gas fue mayor en el pasto Ryegrass que en el pasto Kikuyo, pero la producción de gas total fue mayor en el último.

\section{Referencias bibliográficas}

- Abrego, A. Evaluación de una bacteria ruminal acetogénica en la producción de metano in vitro. Montecillo, Texcoco, Edo. de México. Colegio de postgraduados, 2012. Tesis (Maestría en Ciencias).

- AbuGhazaleh, A. Effect of fish oil and sunflower oil supplementation on milk conjugated linoleic acid content for grazing dairy cows. Animal feed science and technology, v.141, n.3, p.220-32. 2008.

- AOAC. Official methods of analysis. Ed. chemists, Association of official analytical. 16th ed. Gaithersburg, MD, USA: AOAC International, 1999. 
- Arenas, F.A.; Noguera, R.R.; Restrepo, L.F. Effect of different types of fat on the degradation and fermentation kinetics of the dry matter in vitro in ruminants diet.Revista Colombiana de Ciencias Pecuarias, v.23, n.1, p.55-64. 2010.

- Bauman, D.; Baumgard, L.; Corl, B. et al. Biosynthesis of conjugated linoleic acid in ruminants. Journal of Animal Science, v.77, n.E-Suppl, p.1-15. 2000.

- Beauchemin, K.A.; McGinn, S.M.; Benchaar, C. et al. Crushed sunflower, flax, or canola seeds in lactating dairy cow diets: Effects on methane production, rumen fermentation, and milk production. Journal of Dairy Science, v.92, n.5, p.2118-27. 2009.

- Belury, M.A. Dietary conjugated linoleic acid in health: Physiological effects and mechanisms of action. Annual Review of Nutrition, v.22, p.505-31. 2002.

- Bligh, E.G.; Dyer, W.J. A rapid method of total lipid extraction and purification.Canadian Journal of Biochemistry and Physiology, v.37, n.8, p.911-17. 1959.

- Broudiscou, L.; Pochet, S.; Poncet, C. Effect of linseed oil supplementation on feed degradation and microbial synthesis in the rumen of ciliate-free and refaunated sheep.Animal Feed Science and Technology, v.49, n.3-4, p.189-202. 1994.

- Chaucheyras-Durand, F.; Masséglia, S.; Fonty, G. et al. Influence of the composition of the cellulolytic flora on the development of hydrogenotrophic microorganisms, hydrogen utilization, and methane production in the rumens of gnotobiotically reared lambs.Applied and environmental microbiology, v.76, n.24, p.7931-37. 2010.

- Christie, W.W. Gas chromatography and lipids: A practical guide. Ayr, Scotland: Oily Press, 1990.

- Cieslak, A.; Varadyova, Z.; Kisidayova, S. et al. Effect of diets with fruit oils supplements on rumen fermentation parameters, fatty acid composition and methane production in vitro. Journal of Animal and Feed Sciences, v.22, n.1, p.26-34. 2013.

- Corl, B.A.; Baumgard, L.H.; Dwyer, D.A. et al. The role of $\delta 9$-desaturase in the production of cis-9, trans-11 cla. The Journal of Nutritional Biochemistry, v.12, n.11, p.622-30. 2001.

- Correa, H.; Pabón, M.; Carulla, J. Estimación del consumo de materia seca en vacas holstein bajo pastoreo en el trópico alto de Antioquia. Livestock Research for Rural Development, v.21, n.4, 2009. 
- Cosgrove, G.; Waghorn, G.; Anderson, C. et al. The effect of oils fed to sheep on methane production and digestion of ryegrass pasture. Animal Production Science, v.48, n.2, p.189-92. 2008.

- Dilzer, A.; Park, Y. Implication of conjugated linoleic acid (cla) in human health.Critical reviews in food science and nutrition, v.52, n.6, p.488-513. 2012.

- Elghandour, M.M.; Kholif, A.E.; Lopez, S. et al. In vitro gas, methane and carbon dioxide productions of high fibrous diet incubated with fecal inocula from horses fed live yeasts in response to the supplementation with different yeast additives. Journal of Equine Veterinary Science, 2015.

- Estrada, J. Pastos y forrajes para el trópico colombiano. Manizales: Editorial Universidad de Caldas, 2002. 511p.

- Fievez, V.; Dohme, F.; Danneels, M. et al. Fish oils as potent rumen methane inhibitors and associated effects on rumen fermentation in vitro and in vivo. Animal Feed Science and Technology, v.104, n.1-4, p.41-58. 2003.

- Flachowsky, G.; Kamphues, J. Carbon footprints for food of animal origin: What are the most preferable criteria to measure animal yields? Animals, v.2, n.2, p.108-26. 2012.

- García-González, R.; López, S.; Fernández, M. et al. Screening the activity of plants and spices for decreasing ruminal methane production in vitro. Animal Feed Science and Technology, v.147, n.1-3, p.36-52. 2008.

- Gomez-Cortes, P.; de la Fuente, M.A.; Toral, P.G. et al. Effects of different forage: Concentrate ratios in dairy ewe diets supplemented with sunflower oil on animal performance and milk fatty acid profile. Journal of Dairy Science, v.94, n.9, p.457888. 2011.

- Gomez-Cortes, P.; Frutos, P.; Mantecon, A.R. et al. Addition of olive oil to dairy ewe diets: Effect on milk fatty acid profile and animal performance. Journal of Dairy Science, v.91, n.8, p.3119-27. 2008.

- Hervás, G.; Williams, B.A.; Boer, H. et al. Modificación de la fermentación ruminal in vitro de lolium perenne cuando se suplementa con diferentes dosis de grasa. AIDA - IX Jornadas sobre Producción Animal, 2001.

- IPCC. Climate change 2007: The physical science basis. Contribution of working group $i$ to the fourth assessment report of the intergovernmental panel on climate change Cambridge University Press, Cambridge, United Kingdom and New York, NY, USA, 2007. 
- Jalc, D.; Certik, M.; Kundrikova, K. et al. Effect of unsaturated c-18 fatty acids (oleic, linoleic and alpha-linolenic acid) on ruminal fermentation and production of fatty acid isomers in an artificial rumen. Veterinarni Medicina, v.52, n.3, p.87-94. 2007.

- Jayanegara, A.; Kreuzer, M.; Leiber, F. Ruminal disappearance of polyunsaturated fatty acids and appearance of biohydrogenation products when incubating linseed oil with alpine forage plant species in vitro. Livestock Science, v.147, n.1-3, p.104-12. 2012.

- Johnson, K.A.; Johnson, D.E. Methane emissions from cattle. J. Anim Sci., v.73, n.8, p.2483-92. 1995.

- Jordan, E.; Kenny, D.; Hawkins, M. et al. Effect of refined soy oil or whole soybeans on intake, methane output, and performance of young bulls. Journal of Animal Science, v.84, n.9, p.2418-25. 2006.

- Kepler, C.R.; Hirons, K.P.; McNeill, J.J. et al. Intermediates and products of the biohydrogenation of linoleic acid by butyrivibrio fibrisolvens. Journal of Biological Chemistry, v.241, n.6, p.1350-54. 1966.

- Khanal, R.C.; Dhiman, T.R. Biosynthesis of conjugated linoleic acid (cla): A review.Pakistan Journal of Nutrition, v.3, n.2, p.72-81. 2004.

- Li, Y.L.; Meng, Q.X. Effect of different types of fibre supplemented with sunflower oil on ruminal fermentation and production of conjugated linoleic acids in vitro. Archives of Animal Nutrition, v.60, n.5, p.402-11. 2006.

- Lila, Z.A.; Mohammed, N.; Kanda, S. et al. Sarsaponin effects on ruminal fermentation and microbes, methane production, digestibility and blood metabolites in steers. Asian Australasian Journal of Animal Sciences, v.18, n.12, p.1746. 2005.

- López, S.; Dhanoa, M.S.; Dijkstra, J. et al. Some methodological and analytical considerations regarding application of the gas production technique. Animal Feed Science and Technology, v.135, n.1-2, p.139-56. 2007.

- Machmüller, A.; Ossowski, D.A.; Wanner, M. et al. Potential of various fatty feeds to reduce methane release from rumen fermentation in vitro (rusitec). Animal Feed Science and Technology, v.71, n.1-2, p.117-30. 1998.

- McAllister, T.A.; Newbold, C.J. Redirecting rumen fermentation to reduce methanogenesis. Australian Journal of Experimental Agriculture, v.48, n.2, p.7-13. 2008 .

- McCrorie, T.A.; Keaveney, E.M.; Wallace, J.M. et al. Human health effects of conjugated linoleic acid from milk and supplements. Nutrition research reviews, v.24, n.02, p.206-27. 2011. 


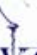

- Mele, M.; Buccioni, A.; Petacchi, F. et al. Effect of forage/concentrate ratio and soybean oil supplementation on milk yield, and composition from sarda ewes. Animal Research, v.55, n.4, p.273-85. 2006.

- Menke, K.H.; Steingass, H. Estimation of the energetic feed value obtained from chemical analysis and in vitro gas production using rumen fluid. Animal Research and Development, v.28, p.7-55. 1988.

- Moss, A.R.; Jouany, J.-P.; Newbold, J. Methane production by ruminants: Its contribution to global warming. Ann. Zootech., v.49, n.3, p.231-53. 2000.

- Palmquist, D.L. Biohydrogenation then and now. European Journal of Lipid Science and Technology, v.109, n.8, p.737-39. 2007.

- Panyakaew, P.; Goel, G.; Lourenço, M. et al. Medium-chain fatty acids from coconut or krabok oil inhibit in vitro rumen methanogenesis and conversion of non-conjugated dienoic biohydrogenation intermediates. Animal Feed Science and Technology, 2013.

- Patra, A.K.; Yu, Z. Effects of coconut and fish oils on ruminal methanogenesis, fermentation, and abundance and diversity of microbial populations in vitro. J Dairy Sci, v.96, n.3, p.1782-92. 2013.

- Preston, T. Tropical animal feeding: A manual for research workers. FAO Animal Productin and Health Paper 126, 1995.

- Prieto-Manrique, E.; Mahecha-Ledesma, L.; Angulo-Arizala, J. et al. Efecto de la suplementación lipídica sobre ácidos grasos en leche de vaca, énfasis en ácido ruménico. Agronomía Mesoamericana, v.27, n.2, p.421-37. 2016a.

- Prieto-Manrique, E.; Vargas-Sánchez, J.; Angulo-Arizala, J. et al. Suplementación con aceite de girasol sobre ácidos grasos de la leche en una lechería tropical. Revista Colombiana de Ciencia Animal-RECIA, v.8, n.Supl, p.297-309. 2016 b.

- Russell, J.B. The importance of $\mathrm{ph}$ in the regulation of ruminal acetate to propionate ratio and methane production in vitro. Journal of Dairy Science, v.81, n.12, p.3222-30. 1998.

- Sánchez, D.E.; Arreaza, L.C.; Abadia, B. Estudio de la cinética de degradación in vitro de cuatro forrajes tropicales y una leguminosa en clima templado. Revista CORPOICA Ciencia y Tecnología Agropecuaria (Colombia), v.6, n.1, p.5868.2005 .

- Soder, K.J.; Brito, A.F.; Rubano, M.D. Short communication: Effect of oilseed supplementation of an herbage diet on ruminal fermentation in continuous culture. $\mathbf{J}$ Dairy Sci, v.96, n.4, p.2551-6. 2013. 
- Theodorou, M.K.; Williams, B.A.; Dhanoa, M.S. et al. A simple gas-production method using a pressure transducer to determine the fermentation kinetics of ruminant feeds. Animal Feed Science and Technology, v.48, n.3-4, p.185-97. 1994.

- Toprak, N.N. Do fats reduce methane emission by ruminants? -a review. Animal Science Papers and Reports, v.33, n.4, p.305-21. 2015.

- Toral, P.G.; Bernard, L.; Belenguer, A. et al. Comparison of ruminal lipid metabolism in dairy cows and goats fed diets supplemented with starch, plant oil, or fish oil. Journal of Dairy Science, v.99, n.1, p.301-16. 2016.

- Van Soest, P.J.; Robertson, J.B.; Lewis, B.A. Methods for dietary fiber, neutral detergent fiber, and nonstarch polysaccharides in relation to animal nutrition. Journal of Dairy Science, v.74, n.10, p.3583-97. 1991.

- Vargas, J.; Cárdenas, E.; Pabón, M. et al. Emisión de metano entérico en rumiantes en pastoreo. Arch. Zootec, v.61, p.51-66. 2012.

- Weiss, M.F.; Martz, F.A.; Lorenzen, C.L. Review: Conjugated linoleic acid: Historical context and implications. The Professional Animal Scientist, v.20, n.2, p.118-26. 2004.

Cómo citar: Vélez-Ruiz, J.P.; Tequin-Ocampo, E.B.; Vargas-Sánchez, J.E. Efecto in vitro del aceite de girasol (Helianthus annuus L.) sobre ácido transvacénico y patrón de ácidos grasos de cadena larga en la digesta ruminal. Revista Veterinaria y Zootecnia, v. 11, n. 1, p. 37-53. DOI: 10.17151/vetzo.2017.11.1.4 